\title{
A Wearable Robotic Exoskeleton for Gait Rehabilitation
}

\author{
Haoyong Yu \\ Department of Biomedical Engineering, National University of Singapore \\ Block E4, \#04-08, 4 Engineering Drive 3 \\ Singapore 117583, Singapore \\ bieyhy@nus.edu.sg
}

\section{Extended Abstract}

Survivors of neurological disorders such as spinal cord injuries, stroke, neurodegenerative diseases or brain injury suffer from serious gait disorders [1]. Physical therapy such as intensive gait training can restore gait performance and improve the quality of life of patients [2]. However, conventional gait training is labour intensive and costly. Rehabilitation robots are able to reduce the labour intensity of therapists and provide better functional outcomes for patients [3].

This paper presents a novel robotic exoskeleton for sub-acute and chronic stroke patients to conduct over-ground gait training at home and outpatient setting. The device is wearable, compact, lightweight and in modular design. It consists of a knee joint and an ankle joint actuated by the same novel series elastic actuator (SEA) [4]. The novel SEA design overcomes the limitation of conventional SEA design by using one low-stiffness translational spring at low speed range and one high-stiffness torsion spring at high speed range. This enables the actuator to have a high intrinsic compliance as well as high peak force. The stiffness of the springs and driving mechanism of the joints are optimized based on the biomechanics of human gait. The robot remains compliant during the majority of the gait cycle and can provide the peak power during push off phase.

We developed a novel human-robot interaction controller to achieve accurate and stable control of the interactive torque between the robot and human joints. Based on the dynamic model of the SEA, the controller provides human joint motion compensation and friction compensation with a disturbance observer [5]. This control method enables the robot to achieve low output impedance when operating in the human-in-charge mode and achieve accurate force tracking when operating in the assistive mode.

During over-ground gait training, it is critical for the robot to synchronize with the human movement and provide the assistance at the right gait phase. We develop a novel control system to synchronize the robot motion with the human motion [6]. Gait events are first detected in real time with a hidden Markov model (HMM). An adaptive oscillator is utilized to estimate the stride percentage of human gait based on the gait event information. Reference trajectories for the robot are then generated with the estimated stride percentage according to a lookup table. Assistive torque is provided to the human joint via an impedance controller according to the synchronous reference gait trajectory and the actual human joint movement.

Extensive experiments have been conducted on healthy subjects to validate the design and evaluate the performance of the design and control of the system. Biomechanical analysis on the gait and muscle activation patterns prove that robot can provide synchronous and effective assistance to the human joints during over-ground walking. Preliminary clinical trials with stroke patients also prove that the system can improve the gait patterns of the patients. More comprehensive clinical trials with randomized control study will be conducted before commercialization of the device.

\section{References}

[1] N. B. Alexander, and A. Goldberg, "Gait disorders: search for multiple causes," Cleveland Clinic journal of medicine, vol. 72, no. 7, pp. 586, 2005.

[2] P. A. Goldie, T. A. Matyas, and O. M. Evans, "Deficit and change in gait velocity during rehabilitation after stroke," Archives of Physical Medicine and Rehabilitation, vol. 77, no. 10, pp. 1074-1082, 1996. 
[3] G. Chen, C. K. Chan, Z. Guo, and H. Yu, "A review of lower extremity assistive robotic exoskeletons in rehabilitation therapy," Critical Reviews ${ }^{\mathrm{TM}}$ in Biomedical Engineering, vol. 41, 2013.

[4] H. Yu, S. Huang, G. Chen, and N. Thakor, "Control design of a novel compliant actuator for rehabilitation robots," Mechatronics, vol. 23, pp. 1072-1083, 2013.

[5] H. Yu, S. Huang, G. Chen, Y. Pan, Z. Guo, "Human-robot interaction control of rehabilitation robots with series elastic actuators," IEEE Trans. on Robotics, vol. 31, no. 5, pp. 1089-1100, 2015.

[6] G. Chen, P. Qi, Z. and H. Yu, "Gait-Event-Based Synchronization Method for Gait Rehabilitation Robots via a Bioinspired Adaptive Oscillator," IEEE Trans. on Biomedical Engineering, in press, 2016. 